\title{
Acoustic Properties of Amorphous Solids at Very Low Temperatures: The Quest for Interacting Tunneling States*
}

\author{
Pablo Esquinazi, Miguel A. Ramos ${ }^{+}$and Reinhard König* \\ Abteilung Supraleitung und Magnetismus, Universität Leipzig, Linnéstrasse 5, \\ D-04103 Leipzig, Germany \\ + Departamento de Física de la Materia Condensada, C-III, Instituto Nicolás \\ Cabrera, Universidad Autónoma de Madrid, E-28049 Madrid, Spain \\ *Leibniz-Institute for Neurobiology, Special Lab Non-Invasive Brain Imaging, \\ Brenneckestrasse 6, D-39118 Magdeburg, Germany
}

We discuss the strain dependence of the acoustic properties of amorphous metals in both normal and superconducting states, in the temperature range $0.1 \mathrm{mK} \leq T \leq 1 \mathrm{~K}$. A crossover is found when the strain energy is of the order of the effective interaction energy between tunneling systems at the corresponding temperature. Our results provide clear evidence for the interaction between tunneling systems, whose energy is in quantitative agreement with theoretical expectations, and reveal that without the knowledge of the corresponding strain dependences, the measured temperature dependences below $\sim 50 \mathrm{mK}$ of the acoustic properties of disordered solids are rather meaningless.

PACS numbers: 62.65.+k, 61.43.Dq, 63.50.+x

\section{INTRODUCTION}

The low temperature properties of amorphous and disordered solids are influenced to a large extent by the interaction of phonons and conduction electrons with tunneling systems (TS). These TS are low-energy excitations whose nature still remains a mystery after thirty years of low-temperature re-

*Published in the special issue "Pushing Physics at Low Temperatures" in honor of Prof. F. de la Cruz. J. Low Temp. Phys. 135, 27 (2004). 


\section{P. Esquinazi, M. Ramos, and R. König}

search. At the beginning of the 80's, Francisco de la Cruz contributed to this research starting successfully in Bariloche with the production of amorphous superconducting ribbons ( $\mathrm{La}_{70} \mathrm{Cu}_{30}$ and $\mathrm{Zr}_{70} \mathrm{Cu}_{30}$ ) by melt spinning using an arc furnace. ${ }^{1}$ With the help of his wife, Maria Elena de la Cruz, Alberto Ridner and one of the authors of this contribution (P.E.), he directed a research work to investigate the influence of heat treatments on the thermal conductivity $\kappa(T)$, among other transport properties, of amorphous superconductors. Well below the critical temperature $T_{c}$, the thermal conductivity is inversely proportional to the density of states $P_{0}$ of the TS, multiplied by the coupling constant between TS and phonons $\gamma$, i.e. $\kappa\left(T<<T_{c}\right) \propto T^{2} /\left(P_{0} \gamma^{2}\right)$. From these data one obtains the product $P_{0} \gamma^{2} \sim 1 \mathrm{~J} / \mathrm{g}$ for amorphous superconductors in the as-quenched state. ${ }^{2}$ This value is of the same order of magnitude as for the amorphous metal $\mathrm{PdSiCu}$ and a factor 5 to 10 smaller than for dielectric glasses. ${ }^{3}$ Low-temperature annealing of the amorphous metal, relaxing but not crystallizing the amorphous structure, leads to an increase of $\kappa\left(T<<T_{c}\right)$ as well as to changes in the acoustic properties. ${ }^{2,4}$ From these as well as from specific heat results it was concluded that the observed changes at the early relaxation states are mostly due to the decrease of the coupling constant $\gamma$ rather than to a change in the density of states of TS (see page $194 \mathrm{ff}$ in Ref. ${ }^{3}$ ).

From measurements done during the last 20 years,${ }^{3}$ we learn that a "relaxed" amorphous structure may show vanishingly small glasslike properties, though still being in the amorphous, disordered state. Since some non amorphous solids show glasslike properties, we conclude that amorphousness is neither a necessary nor a sufficient condition for the existence of glasslike anomalies at low temperatures. Then, the key question is: where does the low-temperature "universality" of glasses rely on? The universality properly refers to an apparent "maximum" value observed in bulk samples for the ratio $C=P_{0} \gamma^{2} /\left(\rho v^{2}\right) \sim 10^{-3}$ (here $\rho$ is the mass density and $v$ the sound velocity of the sample), despite a large variation of all four parameters. The standard tunneling model, based on the assumption of an arbitrary amount of independent, noninteracting tunneling entities with a broad distribution of energy and relaxation time, ${ }^{3}$ does not provide an answer to this experimental fact. These assumptions have been questioned ${ }^{5}$ arguing that the low-energy excitations themselves and the observed universality are indeed the result of interactions between some kind of defects.

The acoustic properties in the $\mathrm{kHz}$ range are an excellent tool to study the interaction between phonons and/or electrons with TS. Following the standard tunneling model ${ }^{6-8}$ (STM), the measurement of the internal friction $Q^{-1}$ and the relative change of sound velocity $\Delta v / v$ with temperature provide information on the density of states of TS $\left(P_{0}\right)$ and their coupling 


\section{Acoustic Properties of Amorphous Solids}

to phonons $(\gamma)$ or conduction electrons $(K)$. Additionally, the relatively low frequencies $(\omega)$ used enable acoustic measurements to be conducted at ultralow temperatures $(T<5 \mathrm{mK})$ as the energy dissipation in the sample $\left(\propto \omega^{3}\right.$ !) can be kept at extremely small levels $\left(<10^{-13} \mathrm{~W}\right)$, thus avoiding self-heating effects. Moreover, this kind of measurement introduces the applied strain $\epsilon$ as a third variable parameter. This parameter shows up as important and fundamental as the temperature to understand the observed deviations of the acoustic properties from the predictions of the STM.

The result we would like to stress in this contribution is that the strain dependence of the acoustic properties of some glasses provides clear evidence for the interaction between TS. The ubiquitous strain dependence shows up as a clue to understand why the temperature dependence of the acoustic properties of glasses below $\sim 50 \mathrm{mK}$ is not understood, in spite of several studies published in the literature during the last decade on this issue. We will show below that there is a clear difference between the strain dependence of the acoustic properties obtained for dielectric and metallic glasses in the superconducting state, in disagreement with the expectations. In this contribution we will deal with similar superconducting alloys as those studied by Francisco de la Cruz $\left(\mathrm{Zr}_{x} \mathrm{Cu}_{1-x}\right),{ }^{1,2}$ but now with critical temperatures below $0.3 \mathrm{~K}$ (for $x=0.3,0.4$ ).

\section{THEORETICAL BACKGROUND}

\subsection{Predictions of the Standard Tunneling Model}

The STM, independently introduced by Anderson, Halperin and Varma, ${ }^{6}$ and Phillips $^{7}$ in 1972, provides a phenomenological description of thermal, dielectric and acoustic properties of amorphous solids below $\sim 1 \mathrm{~K} \cdot{ }^{3,8,9}$ The central idea of the model is the universal existence in glasses of a random distribution $P\left(\Delta_{0}, \Delta\right)=P_{0} / \Delta_{0}$ of independent two-level states or TS with quantum energy splitting $\Delta_{0}$ and asymmetry $\Delta$. The acoustic properties in the $\mathrm{kHz}$ range are much more sensitive than thermal properties to details of the TS and their interactions with phonons and/or electrons. At very-low temperatures and not very-high frequencies $\left(T \ll T_{\mathrm{co}}\right)$, the STM predicts ${ }^{3,8,9}$ for dielectric glasses a relative change of sound velocity $\Delta v / v=C \ln \left(T / T_{0}\right)$ and an internal friction $Q^{-1} \propto C T^{3} / \omega$. Above the crossover temperature $T_{\text {co }}\left(T \gg T_{\text {co }}\right)$, (phonon) relaxational processes add to resonant contributions to the sound velocity and $\Delta v / v=-(C / 2) \ln \left(T / T_{0}\right)$, whereas the relaxation-dominated internal friction reaches a constant "plateau" value $Q^{-1}=(\pi / 2) C . T_{0}$ is an arbitrary reference temperature. In amorphous metals, ${ }^{10}$ conduction electrons provide in addition to phonons an alternative channel for relaxation of the 


\section{P. Esquinazi, M. Ramos, and R. König}

TS, typically dominant below $1 \mathrm{~K}$. In this case, $T_{\text {co }} \sim 1-10 \mu \mathrm{K}$ is estimated instead of $T_{\mathrm{co}} \sim 100 \mathrm{mK}$ as for dielectric glasses, and experiments should therefore show in the whole measured temperature range a soundvelocity variation with both resonant and electron relaxational contributions $\Delta v / v=(C / 2) \ln \left(T / T_{0}\right)$, and an internal friction with a wide plateau.

The first measurements of the acoustic properties of an amorphous superconductor, $\mathrm{Pd}_{30} \mathrm{Zr}_{70}$, in the superconducting and normal state, were performed by Neckel et al. ${ }^{11}$ Well below its transition temperature $T \ll T_{\mathrm{c}}$, an amorphous superconductor was expected to behave as a dielectric glass. However, this and subsequent experiments of the same kind ${ }^{3}$ have shown that the interaction mechanisms between TS and conduction electrons are still far from being well understood.

Although some experiments ${ }^{3,8,9,12}$ have given support to the STM, significant discrepancies below $\sim 100 \mathrm{mK}$ have been reported more recently: The internal friction in dielectric glasses decreases for $T<T_{\text {co }}$ slower than $Q^{-1} \propto T^{3}, 3,13,14$ the slopes of $\Delta v / v$ vs. $T$ sometimes differ from the predicted values, and the acoustic properties of both dielectric and metallic glasses exhibit a strong, unexpected strain dependence. ${ }^{3,13,15}$

Some of the observed strain dependences can be accounted for by including the strain energy in the population of the energy levels in equilibrium, i.e.

$$
n_{0} \propto \tanh \left(\sqrt{\Delta_{0}^{2}+\left(\Delta+2 \gamma \epsilon_{0} \sin (\omega t)\right)^{2}} / 2 k_{\mathrm{B}} T\right)
$$

where $\epsilon_{0}$ is the amplitude of the stress field. ${ }^{3}$ Numerical results obtained with values of the strain $\epsilon_{0}$ similar to those used in experiments with $\mathrm{SiO}_{2}$ resemble partially some of the measured features ${ }^{3}$ namely: (1) For strain energies of the order of the thermal energy $\gamma \epsilon_{0} \sim k_{\mathrm{B}} T$ the sound velocity decreases with strain and shows a higher slope with $T$ at $T<T_{\mathrm{co}}$, (2) the position of its maximum at $T_{\text {co }}$ shifts to higher temperatures with strain, and (3) at $T \ll T_{\text {co }}$ the sound velocity saturates. The internal friction, on the other hand, is not affected by the strain at $\epsilon_{0} \leq 10^{-7}$, whereas it appears to decrease slightly at higher strains. ${ }^{13} \mathrm{~A}$ more rigorous approach has been done in Ref. ${ }^{16}$ and the results resemble those obtained using the simple assumption of a population change. In the limit of slow relaxation of the TS's at $T \ll T_{\text {co }}$, analytical results were also obtained for the change of $Q^{-1}$ with strain. ${ }^{3,16}$

We note that Stockburger et al. ${ }^{16,17}$ developed a theory based on the tunneling model, which accounted for a strain dependence of the sound velocity and the internal friction of amorphous metals ${ }^{17}$ and dielectric glasses, ${ }^{16}$ in terms of an intrinsically nonlinear response of the TS to an external disturbance. This saturation regime is achieved when $\gamma \epsilon_{0}>k_{\mathrm{B}} T$. In this work, however, we will deal always with applied strains orders of magnitude below 


\section{Acoustic Properties of Amorphous Solids}

this saturation limit.

\subsection{Ensemble of Interacting Tunneling Systems}

As pointed out above, one striking feature of disordered solids is the universality of the maximum value of the plateau observed in the internal friction of bulk dielectric glasses $Q_{\text {plateau }}^{-1} \sim 10^{-3}$, despite a great variation of the parameters involved. As a possible reason for this fact, Yu and Leggett ${ }^{5}$ argued that this may be the result of interactions between some kind of defects. On general grounds, ${ }^{5}$ the effective interaction between two TS separated by a distance $r$ is dipolar elastic and the interaction energy reads $\sim U_{0} / r^{3}$, with $U_{0} \approx \gamma^{2} / \rho v^{2}$. The observed universality of low-temperature glassy properties can therefore be written in terms of the dimensionless interaction strength as $P_{0} U_{0} \approx$ const $\approx C \sim 10^{-3} \cdot{ }^{18}$

In a recent paper, Burin et al. ${ }^{19}$ considered the delocalization of lowenergy excitations in disordered systems due to an external alternating acoustic or electric field. The delocalization depends on both the strain amplitude and the distance between resonant TS neighbors, which depends on the elastic interaction $U_{0}$ between them. Similarly to Eq. (1) the asymmetry $\Delta$ becomes strain and time dependent and may become smaller than $\Delta_{0}$ for large enough strain amplitudes. In this case, they argue that for sufficiently small frequencies compatible with our acoustic frequencies, the strain increases the effective number of delocalized TS, increasing $Q^{-1}$ below $T_{\text {co }}$ due to a shift in the crossover condition $\omega \tau<1$ to lower temperatures, $\tau$ being the characteristic relaxation time of the TS. Recently done measurements of the dielectric constant of an insulating glass as a function of electric field and thickness apparently show agreement with these theoretical predictions. ${ }^{20}$ However, the predicted changes due to delocalization with strain appear to be at odds with the changes with strain observed in the sound velocity and internal friction of $\mathrm{SiO}_{2} \cdot{ }^{13}$ We will see in the next section that the changes with strain observed in amorphous metals also disagree with these predictions. We will consider and discuss the role of the strain energy within the model of interacting TS pairs using a different point of view in the next section, after presenting our experimental results.

\section{EXPERIMENTAL RESULTS AND DISCUSSION}

Ribbons of amorphous $\mathrm{Zr}_{\mathrm{x}} \mathrm{Cu}_{1-\mathrm{x}}(\sim 30 \mu \mathrm{m}$ thick $)$ were prepared with the melt-spinning technique in an argon-controlled environment similar to that described in Ref. ${ }^{1}$ Young-modulus sound velocity variation and acoustic attenuation were measured at around $1 \mathrm{kHz}$ using the vibrating-reed technique $^{3,12}$ in a nuclear adiabatic demagnetization cryostat. ${ }^{21}$ The mea- 


\section{P. Esquinazi, M. Ramos, and R. König}

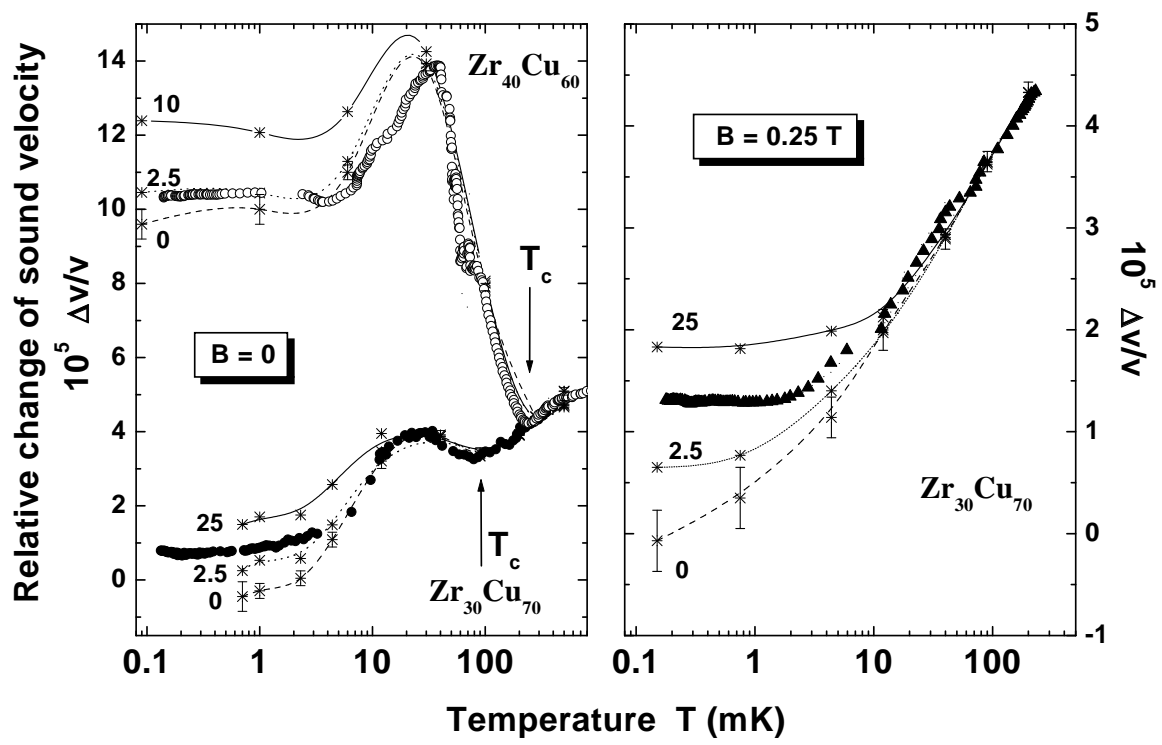

Fig. 1. Left panel: Sound-velocity variation of amorphous superconductors $\mathrm{Zr}_{30} \mathrm{Cu}_{70}$ (solid symbols) and $\mathrm{Zr}_{40} \mathrm{Cu}_{60}$ (open symbols), measured at $1 \mathrm{kHz}$ with a driving voltage of $1 \mathrm{~V}$. The corresponding superconducting transition temperatures $T_{c}$ are indicated by arrows. Right panel: Soundvelocity variation of $\mathrm{Zr}_{30} \mathrm{Cu}_{70}$ in normal metallic state at a driving voltage of $2 \mathrm{~V}$. In all cases, extrapolated data to zero strain and interpolated data for constant-strain curves (see text) are also shown, with lines as eyeguides: labels indicate the corresponding strains in units of $10^{-10}$.

surements were made as a function of temperature $(0.1 \mathrm{mK} \leq T \leq 1 \mathrm{~K})$, both in the superconducting and normal state of the samples $\left(T_{c} \simeq 280 \mathrm{mK}\right.$ and $95 \mathrm{mK}$ for $x=0.4$ and 0.3 , respectively), and also as a function of the applied strain $\left(4 \times 10^{-11} \lesssim \epsilon \lesssim 10^{-8}\right)$ at constant temperatures. For the measurements as a function of temperature, the driving voltage remained constant (and not the applied strain).

In order to study the effects of the applied strain in greater detail, we conducted $^{22}$ complementary experiments by carefully measuring, at selected constant temperatures, the full resonance curves for several given excitation voltages, obtaining the corresponding applied strain from the reed vibration amplitude. ${ }^{15}$ It is important to stress that each plot of $\Delta v / v$ and $Q^{-1}$ as a function of strain consists of up to 30 data points, each of which is the result of a single resonance curve measured in average in one hour. This implies that the temperature had to be kept constant for at least $24 \mathrm{~h}$ even at the 


\section{Acoustic Properties of Amorphous Solids}

lowest temperatures, far below $1 \mathrm{mK}$. Following this procedure, we were able to obtain curves of as a function of the applied strain (see Figs. 4 and 5 in Ref. ${ }^{15}$ for $\mathrm{PdSiCu}$ and Fig. 2 in Ref. ${ }^{22}$ for $\mathrm{Zr}_{30} \mathrm{Cu}_{70}$ ) for several selected temperatures. By interpolating those curves we can obtain true constantstrain curves of the acoustic properties as a function of temperature, and even their presumed very-low-strain behavior by extrapolating them to zero.

In Fig. 1 and Fig. 2, we show $\Delta v / v$ and $Q^{-1}$ data, respectively, for $\mathrm{Zr}_{30} \mathrm{Cu}_{70}$, in both superconducting (SC) and normal (N, with a magnetic field $B=0.25 \mathrm{~T}$, which is above the critical field) states, obtained at a given excitation voltage of the reed during warm-up periods of the nuclear stage. Similar data were obtained for $\mathrm{Zr}_{40} \mathrm{Cu}_{60}$ (with $B=0$ ), ${ }^{22,23}$ that are also shown in the left panels of Figs. 1 and 2 , as well as for the metallic glass PdSiCu. ${ }^{15}$ Within the STM, the maximum in $\Delta v / v$ at $T_{\text {co }} \approx 30 \mathrm{mK}$ and the corresponding decrease of $Q^{-1}$ with decreasing temperature should originate from the abovementioned crossover, occurring when $\omega \tau \sim 1$. As expected, this peak disappears in the normal metallic state, since this crossover shifts to orders of magnitude lower temperatures due to the much faster relaxation rate of TS by electrons than by phonons.

However, at lower temperatures both $\Delta v / v$ and $Q^{-1}$ strongly depend on the excitation voltage (hence on strain), a behavior similar to that observed in the metallic glass $\mathrm{PdSiCu} .{ }^{13,15}$ The sound velocity clearly increases and the internal friction decreases with increasing voltage or strain, and furthermore they do not exhibit in any case below $\approx 10 \mathrm{mK}$ the behavior predicted by the STM. Moreover, we remind that the STM is linear in strain and cannot account for any strain dependence of acoustic properties. Some of the constant-strain curves are also shown in Fig. 1 and Fig. 2. We note that the interpolated constant strain curves for $\epsilon>10^{-10}$ confirm the main temperature dependence observed from direct constant-voltage curves.

The following facts are interesting to note: (a) The apparent crossover at $T_{\text {co }} \sim 30 \mathrm{mK}$ observed in $\mathrm{Zr}_{30} \mathrm{Cu}_{70}$ in the superconducting state is an artifact due to the applied strain. The internal friction does not start to decrease at this temperature but it remains in first approximation independent of $T$ for low enough strains. (b) The conduction electrons influence both acoustic properties and produce the anomaly at $T_{c}$, clearly recognized after the application of the magnetic field and in good agreement with previous measurements at higher temperatures. ${ }^{4,11}$ However, two new results are obtained from the strain dependence: (c) At $T<2 \mathrm{mK}$ the sound velocity shows similar $T$-dependence both in the normal and superconducting state. (d) The strain dependence of the acoustic properties (in particular $Q^{-1}$ ) depends on the state of the sample. This, as well as the results to be presented in Fig. 3, indicate that the conduction electrons influence the 


\section{P. Esquinazi, M. Ramos, and R. König}

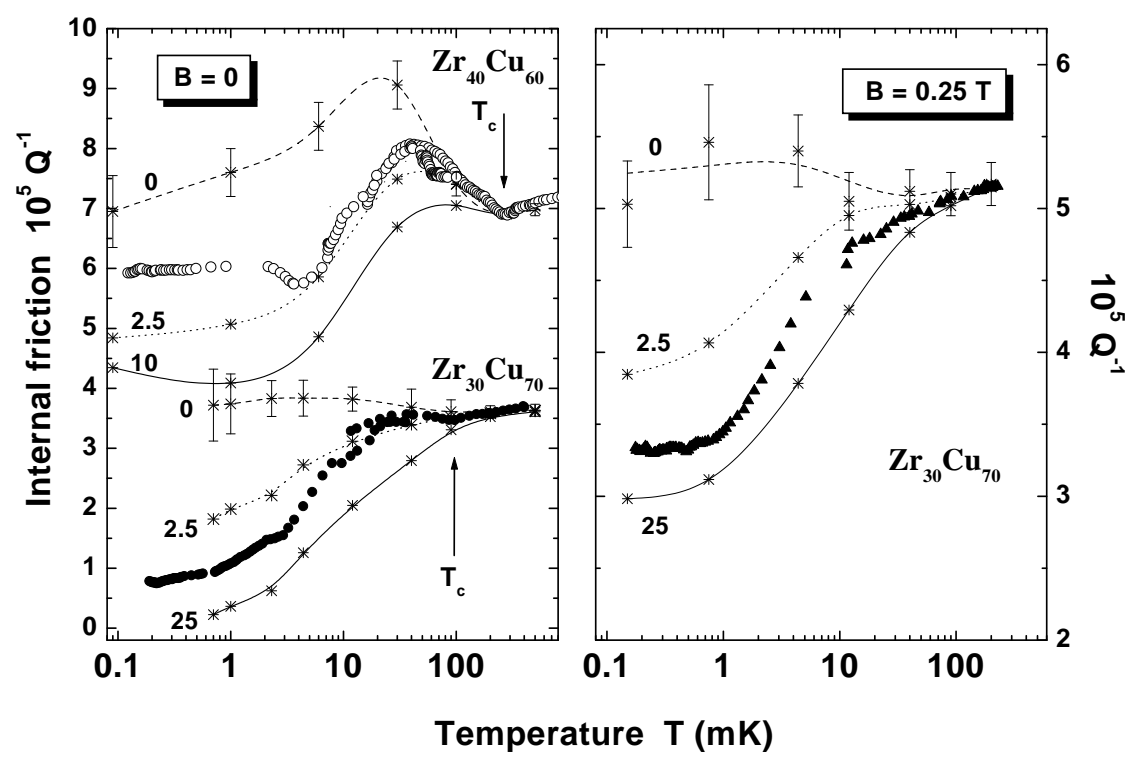

Fig. 2. Left panel: Internal friction of amorphous superconductors $\mathrm{Zr}_{30} \mathrm{Cu}_{70}$ (solid symbols) and $\mathrm{Zr}_{40} \mathrm{Cu}_{60}$ (open symbols), measured at $1 \mathrm{kHz}$ with a driving voltage of $1 \mathrm{~V}$. The corresponding superconducting transition temperatures $T_{c}$ are indicated by arrows. For clarity the data for $\mathrm{Zr}_{30} \mathrm{Cu}_{70}$ have been shifted vertically by $-1.5 \times 10^{-5}$. Right panel: Internal friction of $\mathrm{Zr}_{30} \mathrm{Cu}_{70}$ in normal metallic state at a driving voltage of $2 \mathrm{~V}$. In all cases, extrapolated data to zero strain and interpolated data for constant-strain curves (see text) are also shown, with lines as eyeguides: labels indicate the corresponding strains in units of $10^{-10}$.

strain dependence. ${ }^{22}$ All these results cannot be explained within the STM.

In Fig. 3, all sets of constant-temperature measurements for $\mathrm{Zr}_{30} \mathrm{Cu}_{70}$ are plotted as a function of the dimensionless ratio of strain energy to thermal energy, $\gamma \epsilon / k_{\mathrm{B}} T$, along almost five orders of magnitude. A typical value of $\gamma=1 \mathrm{eV}$ has been used for the coupling constant. As can be observed, all curves show the same $Q^{-1}$ "plateau" value at $\gamma \epsilon / k_{\mathrm{B}} T<10^{-4}$ (strikingly, also in the $\mathrm{SC}$, dielectric case). Moderately increasing the strain, so that $\gamma \epsilon / k_{\mathrm{B}} T>10^{-4}$, makes $Q^{-1}$ to decrease logarithmically with $\gamma \epsilon / k_{\mathrm{B}} T$ (the slope of the solid line shown in Fig. 3 is $\left.\log \left(\gamma \epsilon / k_{\mathrm{B}} T\right) / 3\right)$ for the SC case. When conduction electrons contribute to the relaxation of TS in the whole experimental temperature range, the same behavior is observed, though the slope is less steep $\left(\log \left(\gamma \epsilon / k_{\mathrm{B}} T\right) / 5\right)$. The difficulties encountered in zero- 


\section{Acoustic Properties of Amorphous Solids}

strain extrapolations at the lowest temperatures (say, below $5-10 \mathrm{mK}$ ) is also made clearer now: even the very low strains used here are not low enough to completely reach the "plateau" limit in some cases. On the other hand, $\Delta v / v$, which is rather governed by resonant processes (especially in the SC state), also exhibit a crossover at $\gamma \epsilon / k_{\mathrm{B}} T \sim 10^{-4}$ from the strainindependent or linear behavior into a regime of strong increase of the sound velocity with strain, either in N or SC states. Furthermore, although we have shown and discussed here only the case of $\mathrm{Zr}_{30} \mathrm{Cu}_{70}$, a very similar plot ${ }^{22}$ can be obtained for $\mathrm{Zr}_{40} \mathrm{Cu}_{60}$ data in the $\mathrm{SC}$ state, and also for the normal metal $\mathrm{PdSiCu}$, pointing once again to a universal behavior of glasses.

We want to emphasize that in all cases the crossover from strainindependent to strain-dependent behavior occurs when the above defined ratio is $\gamma \epsilon / k_{\mathrm{B}} T \approx 2-5 Q_{\text {plateau }}^{-1} \approx P_{0} U_{0}$. Therefore, one can make the following argumentation within the interacting model: $P_{0} \times k_{\mathrm{B}} T$ is the number of thermally activated TS per unit volume, then $P_{0} U_{0} \times k_{\mathrm{B}} T$ is the total effective interaction energy between TS at the considered temperature. We thus have two limits taking into account the strain energy:

(i) For $\gamma \epsilon \ll\left(P_{0} U_{0} \times k_{\mathrm{B}} T\right)$, i.e. when the strain energy driven by the external sound wave is less than the interaction energy between TS, one may observe the "true" unperturbed response of the TS, in which only their interaction and the thermal energy determine the temperature dependence of the acoustic properties. Since in most of the experiments performed in the past the strain energy was not considered, we do not know a priori if the STM applies at this limit. Going a step further, we may speculate that in this regime the TS are delocalized in the sense that their interaction energy overwhelms all other perturbations (thermal and of the applied strain) and coherent behavior is expected. In this case we may have the maximum possible density of TS as scattering centers for phonons.

(ii) When $\gamma \epsilon \gg\left(P_{0} U_{0} \times k_{\mathrm{B}} T\right)$, the system is perturbed and nonlinear effects appear in the acoustic properties. In this case the linear STM picture of independent TS is surely no longer valid. Moreover, we expect that the strain energy influences the coupling between TS in such a way that an effective localization and reduction of the density of states may arise, since a "localized" TS has less probability to scatter resonantly a phonon than in the case of an ensemble of TS with a broader spectrum of energies and relaxation times.

In other words, the ratio $\gamma \epsilon /\left(k_{\mathrm{B}} T\right) \approx P_{0} U_{0} \sim Q_{\text {plateau }}^{-1}$ would therefore indicate the border between a linear and a nonlinear regime given by the competition between interaction and perturbation energies. This crossover will occur around $\sim 20-50 \mathrm{mK}$ for typical experiments. Strictly speaking, it depends on the variable $\gamma \epsilon / k_{\mathrm{B}} T$, since strain and thermal energies play 
P. Esquinazi, M. Ramos, and R. König

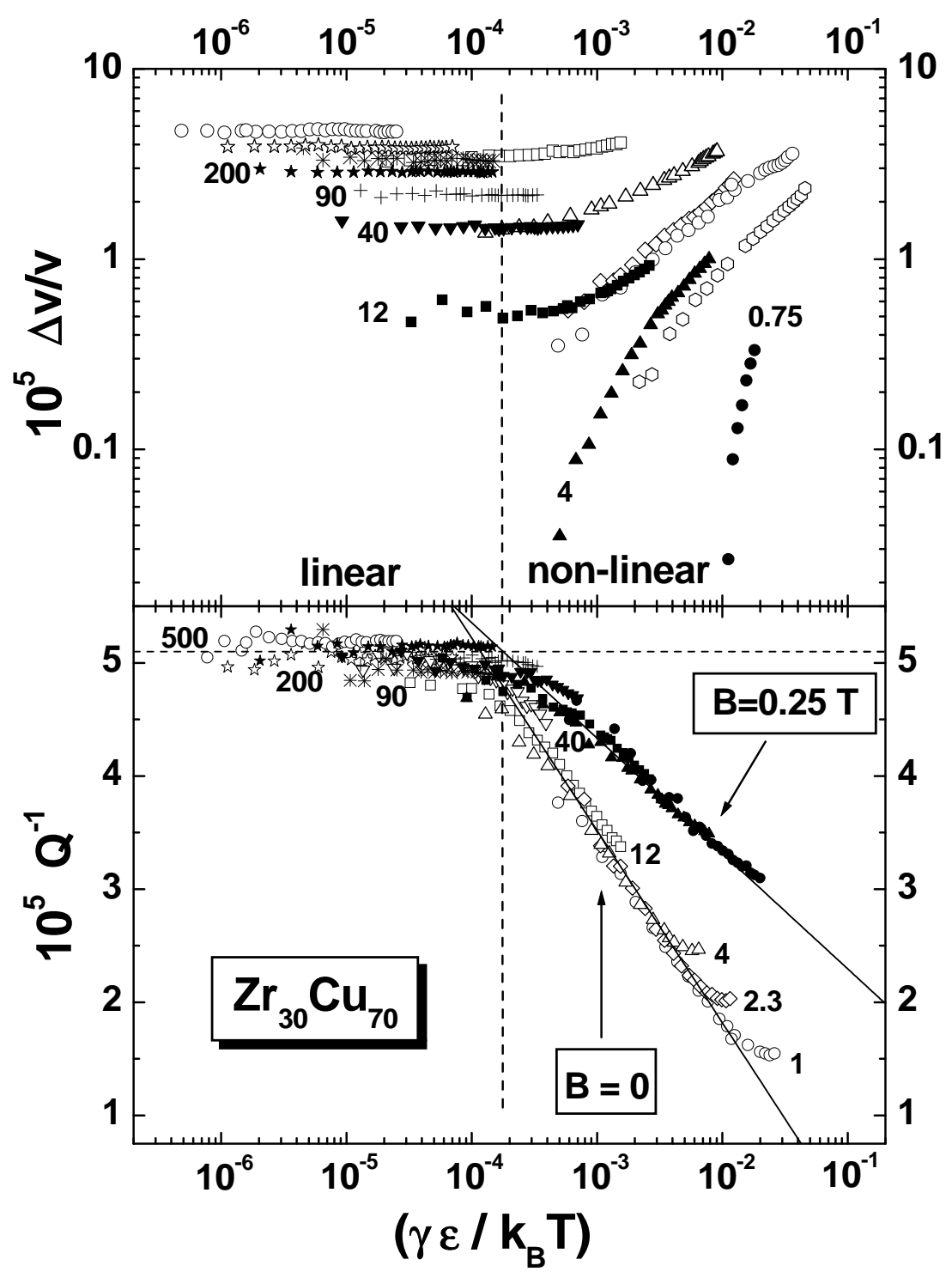

Fig. 3. Sound-velocity variation $\Delta v / v$ (top) and internal friction $Q^{-1}$ (bottom) measured for $\mathrm{Zr}_{30} \mathrm{Cu}_{70}$ (open symbols, $B=0$; solid symbols, $B=0.25$ $\mathrm{T}$ ), plotted versus the ratio of strain energy to thermal energy in a logarithmic scale, for several sets of constant-temperature data which are labelled in $\mathrm{mK}$. Solid lines show the linear decrease of $Q^{-1}$ with $\log \left(\gamma \epsilon /\left(k_{\mathrm{B}} T\right)\right)$ above the crossover at $\gamma \epsilon /\left(k_{\mathrm{B}} T\right) \approx P_{0} U_{0}$. 


\section{Acoustic Properties of Amorphous Solids}

an inextricably combined role in the acoustic properties of glasses at low temperatures due to the mutual interaction of TS.

Interestingly, the reported behavior on the acoustic properties of these metallic glasses, either in $\mathrm{N}$ or $\mathrm{SC}$ state, as a function of the strain, is very different from that found ${ }^{13}$ in vitreous silica, $\mathrm{v}-\mathrm{SiO}_{2}$, where the sound velocity decreases and the maximum in $\Delta v / v$ shifts to higher temperatures with increasing strain $\epsilon$ varying from $2 \times 10^{-8}$ to $10^{-6}$, whereas the internal friction $Q^{-1}$ exhibits a negligible strain dependence. Since in our work on metallic glasses we have employed a very low strain range (at least two orders of magnitude lower than those used in the $\mathrm{SiO}_{2}$ experiments), it might be that the aforementioned effect of the strain energy changing the thermal population of the energy levels in equilibrium (see Eq. (1)) is dominant in that higher strain range used for $\mathrm{v}-\mathrm{SiO}_{2}$. In addition, at the highest applied strains for the lowest temperatures measured in $\mathrm{Zr}_{\mathrm{x}} \mathrm{Cu}_{1-\mathrm{x}}$, i.e. for $\gamma \epsilon / k_{\mathrm{B}} T>10^{-2}$ (see Fig. 3), the logarithmic decrease of the internal friction with increasing $\gamma \epsilon / k_{\mathrm{B}} T$ seems ceasing to hold. So, both effects altogether could explain the observed strain-dependence behavior of $\mathrm{v}-\mathrm{SiO}_{2}$. Another speculative possibility is that vitreous silica exhibits indeed a behavior as a function of strain opposite to other glasses, as it does in other physical properties due to its particular tetrahedral network.

In summary, we have presented a detailed study of the acoustic properties of some metallic glasses in the temperature range $0.1 \mathrm{mK} \leq T \leq 1 \mathrm{~K}$, in both $\mathrm{N}$ and $\mathrm{SC}$ states. The main experimental finding has been a general, strong strain dependence of the acoustic properties of glasses observed at very low temperatures in all the studied cases, for both internal friction and sound velocity variation. In the normal metallic state, sound-velocity variation $\Delta v / v$ and internal friction $Q^{-1}$ seem to recover the expected STM behavior in the zero-strain limit. Nevertheless, in the superconducting state the plateau in $Q^{-1}$ also extends down to the lowest temperatures $(T<1 \mathrm{mK})$ in the zero-strain limit instead of exhibiting the expected $\propto T^{3}$ dependence, predicted by the STM.

By deeper analyzing all these data, we have found a general crossover from a linear to a non-linear regime with increasing the ratio of strain over thermal energy. In fact, this crossover occurs always when $\gamma \epsilon / k_{\mathrm{B}} T \approx P_{0} U_{0}$, in agreement with the interaction energy postulated by $\mathrm{Yu}$ and Leggett, ${ }^{5}$ and by Burin and Kagan. ${ }^{18}$ Furthermore, our results show that the measured $T$-dependences of the acoustic properties of disordered materials below $\sim 50 \mathrm{mK}$ are rather meaningless, unless one measures the corresponding strain dependences too. We have also shown that none of the current models in the literature are able to account for the temperature- and straindependent behavior of the acoustic properties of amorphous metals in the 


\section{P. Esquinazi, M. Ramos, and R. König}

superconducting state at very low temperatures.

\section{ACKNOWLEDGMENTS}

This research was supported by the DFG, DAAD and EU Contract ERB FMGE CT950072. Fruitful discussions with many participants of the Workshop on Collective Phenomena in the Low-Temperature Physics of Glasses (Dresden, 2003) are gratefully acknowledged.

\section{REFERENCES}

1. H. Tutzauer, P. Esquinazi, M. E. de la Cruz and F. de la Cruz, Rev. Sci. Instruments 51, 546 (1980).

2. P. Esquinazi, M. E. de la Cruz, A. Ridner and F. de la Cruz, Solid State Commun. 44, 941 (1982).

3. For a recent review see: P. Esquinazi, ed.: Tunneling Systems in Amorphous and Crystalline Solids (Springer, Berlin, 1998).

4. P. Esquinazi and J. Luzuriaga, Phys. Rev. B 37, 7819 (1988).

5. C. C. Yu and A. J. Leggett, Comments Cond. Mat. Phys. 14, 231 (1988).

6. P. W. Anderson, B. I. Halperin, and C. M. Varma, Phil. Mag. 25, 1 (1972).

7. W. A. Phillips, J. Low Temp. Phys. 7, 351 (1972).

8. W. A. Phillips, ed.: Amorphous Solids: Low Temperature Properties (Springer, Berlin, 1981); W. A. Phillips, Rep. Prog. Phys. 50, 1657 (1987).

9. S. Hunklinger and A. K. Raychaudhuri, in Progress in Low Temperature Physics, Vol. IX, edited by D.F. Brewer (Elsevier, Amsterdam, 1986).

10. J.L. Black, in Glassy Metals I, edited by H.-J. Güntherodt and H. Beck (Springer, Berlin, 1981).

11. H. Neckel. P. Esquinazi, G. Weiss and S. Hunklinger, Solid State Commun. 57, 151 (1986).

12. A. K. Raychaudhuri and S. Hunklinger, Z. Phys. B 57, 113 (1984).

13. P. Esquinazi, R. König, and F. Pobell, Z. Phys. B 87, 305 (1992).

14. J. Classen, T. Burkert, C. Enss, and S. Hunklinger, Phys. Rev. Lett. 84, 2176 (2000).

15. M. A. Ramos, R. König, E. Gaganidze, and P. Esquinazi, Phys. Rev. B 61, 1059 (2000).

16. J. T. Stockburger, M. Grifoni, and M. Sasseti, Phys. Rev. B 51, 2835 (1995).

17. J. Stockburger, M. Grifoni, M. Sassetti, and U. Weiss, Z. Phys. B 94, 447 (1994).

18. A. L. Burin, D. Natelson, D. D. Osheroff, and Y. Kagan, in Ref., ${ }^{3}$ Chap. 5.

19. A. L. Burin, Y. Kagan, and I. Y. Polishchuk, Phys. Rev. Lett. 86, 5616 (2001).

20. F. Ladieu, J. Le Cochec, P. Pari, P. Trouslard, and P. Ailloud, Phys. Rev. Lett. 90, 205501 (2003).

21. K. Gloos, P. Smeibidl, C. Kennedy, A. Singsaas, P. Sekowski, R. Mueller and F. Pobell, J. Low Temp. Phys. 73, 101 (1988). 


\section{Acoustic Properties of Amorphous Solids}

22. R. König, M. A. Ramos, I. Usherov-Marshak, J. Arcas-Guijarro, A. HernandoMañeru, and P. Esquinazi, Phys. Rev. B 65, 180201(R) (2002).

23. R. König, I. Usherov-Marshak, M. A. Ramos, P. Esquinazi, J. Arcas-Guijarro, and A. Hernando-Mañeru, Physica B 316-317, 509 (2002). 